\title{
Implementation of Data Compression Techniques in Mobile Ad hoc Networks
}

\author{
B.Ruxanayasmin \\ Student, M.Tech (DECS) \\ Gudlavalleru Engineering \\ College \\ Gudlavalleru, Krishna Dt, AP, \\ India
}

\author{
B.Ananda Krishna \\ Professor \\ Department of ECE \\ Gudlavalleru Engineering \\ College \\ Gudlavalleru, Krishna Dt, AP, \\ India
}

\author{
T.Subhashini \\ Assistant Professor \\ Department of ECE \\ Gudlavalleru Engineering \\ College \\ Gudlavalleru, Krishna Dt, AP, \\ India
}

\begin{abstract}
Mobile Ad hoc Networks (MANETs) are wireless infrastructure less networks can be easily formed or deployed due to its simple infrastructure. Since each node acts as a router, the nodes must assist in discovery and maintain the network routes for long standing which is not possible due to limited battery source. This reflects on link failures which increase the overhead. Since the MANETs has tremendous applications in commercial, military, mobile conferencing outside the office, battlefield communications, embedded sensor devices that automate household functions, etc., the goal is to maintain the network sustain for long by reducing power consumption. The power consumption plays a vital role in MANETS, and there are lots of low power consumption techniques were designed. Among many, data compression technique is a simple technique, with the benefit of reducing the transmission rate that consumes less bandwidth and low power. Hence in this work, Lempel - Ziv - Welch (LZW) compression algorithm is implemented and the simulation results proved that the data can be transmit with low power consumption without any loss of data. This, in turn helps in reducing the battery consumption, thereby increasing the battery life.
\end{abstract}

\section{Keywords}

MANET, power consumption, link failures, compression, decompression.

\section{INTRODUCTION}

A Mobile Ad hoc Network is an autonomous system consisting of mobile routers connected by wireless links which forms an arbitrary graph [1]. The routers are free to move randomly and organize themselves arbitrarily due to which the network's topology may change unpredictably. The applications of ad hoc networks are personal area networking, military environments, civilian environments, emergency operations. And the limitations of ad hoc networks are restricted wireless transmission range, broadcast nature of the wireless medium, hidden terminal problem, packet losses due to transmission errors, mobility induced route changes and packet losses, battery constraints, potentially frequent network partitions, etc.

In these inadequacies, limited battery life is one of the main constraints and there are many solutions proposed to improve the battery life. Some of them are

1.1 Minimizing power by reducing link failures

1.2 Specially designed power control techniques

1.3 By modifying cryptographic algorithms

1.4 Data compression techniques

\subsection{Minimizing Power by Reducing Link Failures}

Most of the wired networks rely on the symmetric links which are always fixed. But this is not a case with Ad hoc networks as the nodes are mobile and constantly changes their positions within network results link breakages. On the other hand, when a node (sender) fails to transmit data to its next-hop receiver for a certain period of time, the node simply assumes that the link is broken. The source node is thus notified to discover a new route to the destination based on the assumption that the link failure is due to user mobility and node failure, and so forth. However, a link failure event can be caused not only by user mobility and node failure, but also by link-layer contention. The latter case is also referred to as the false link failure, where the intermediate node that fails to relay the data will also inform the source node to discover a new route by mistakenly assuming that the link is broken. The route discovering procedure is very time consuming and imposes enormous overhead to the network and the entire process requires massive power. Thus, there is a need to minimize the link failures in MANETs to improve the efficiency of the network by reducing overhead, power, etc.

AODV has been proposed by IETF (Internet Engineering Task Force) which provides local repair to recover the route when a link break in an active route occurs. But local repair is only performed when a node has already detected broken link and the detection consumes too much time. There is another proposal in which the node listens in all frames including data packets and routing control packet to maintain local route cache. The fast route discovery and local recovery is achieved by local route cache when a broken link occurs [2]. The drawback of this scheme is that nodes maintain a backup route which consumes additional energy.

\subsection{Specially Designed Power Control Techniques}

Nodes allows to modify their transmit power to increase network capacity and reduce energy consumption. This suggests that a route with a large number of low-power hops may be more energy-efficient than one with fewer high-power hops. A route that contains more hops may experience a higher probability of network route failure or link-layer packet retransmission. When evaluating the effectiveness of powercontrol techniques, it is important to consider whether the energy consumption model is realistic, particularly in its treatment of receiving. The two subsections of power control techniques are the topology control and minimum energy routing. The topology control is to assign for node transmit 
power that minimize the maximum transmit power used in the network. In minimum energy routing it reduces power per packet transmission and the sleep management reduces idle power through duty cycle [3].

\subsection{Modification of Cryptographic Algorithms}

Mobile nodes able to roam independently make them easier to be captured, compromised and hijacked. Since tracking down a particular mobile node in a large-scale ad hoc network could be hard and attacks by a compromised node from within the network are more harmful and much harder to detect and moreover creating and maintaining trust among peer nodes is difficult. The security problems in the network can be overcome by implementing strong cryptographic algorithms. But these algorithms consume a significant amount of computing resources such as CPU time, memory, and battery power. CPU and memory usability are increasing with a suitable rates, but battery technology is increasing at slower rate which forms "battery gap". The design of efficient secure protocols for wireless devices from the view of battery consumption needs to understand how encryption techniques affect the consumption of battery power in data transmission [4].

\subsection{Data Compression Technique}

Another important and simple technique for reducing power consumption is Data Compression, which consumes less power by transmitting compressed data results increasing in battery life. A compression algorithm is used to convert data from an easy-to-use format to one optimized for compactness. Due to compression, the number of bits can be reduced to maximum extend so that the need of memory and bandwidth are very less. Also, the compressed text resembles a scramble message and an attacker in middle cannot able to understand. Therefore, the data compression not only reduces the size of the original text, but also gives data security.

Likewise, a decompression program returns the information to its original form. The data compression algorithms are classified into lossless and lossy. A lossless technique is that the restored data file is identical to the original. This is absolutely necessary for many types of data, for example: executable code, word processing files, tabulated numbers etc. The compression methods used for images and sounds are lossy, as they will be degraded in comparison with the original files.

When compared different lossless compression algorithms, it is observed that the LZW compression algorithm has higher compression ratio than other coding techniques as shown in table1.

Table1. Comparison of lossless compression techniques

\begin{tabular}{|c|c|c|c|}
\hline $\begin{array}{c}\text { Types of } \\
\text { file }\end{array}$ & $\begin{array}{c}\text { Huffman } \\
\text { Encoding }\end{array}$ & $\begin{array}{c}\text { LZW } \\
\text { Compression }\end{array}$ & $\begin{array}{c}\text { Run } \\
\text { Length } \\
\text { Encoding }\end{array}$ \\
\hline Text file & $34 \%$ & $56 \%$ & $25 \%$ \\
\hline Speech file & $35 \%$ & $36 \%$ & $27 \%$ \\
\hline Image file & $6 \%$ & $12 \%$ & $3 \%$ \\
\hline \multicolumn{3}{|c|}{ Percentage of Compression } \\
\hline
\end{tabular}

In this work, Lempel-Ziv-Welch (LZW) compression algorithm is implemented, which is fast and simple to apply and works best for files containing lots of repetitive data. LZW algorithm is efficient because the output resembles numerical data and also it doesn't need to pass the string table to the decompression code. The table can be recreated as it was during compression, using the input stream as data. This avoids insertion of large string translation table with the compressed data and it also provides a better compression ratio (maximum of 5:1). The LZW compression can be used in a variety of file formats: TIFF files, GIF files, Bitmap files, all kind of text files.

Section 2 describes the related work on power consumption and Analysis of existing algorithms is given in section 3 . Overview of proposed algorithm is given in section 4 and implementation and simulation results in section 5 and conclusion and future scope in section 6 .

\section{RELATED WORK}

The authors Subir kumar sharkar, T.G.Basavaraju, C.Puttamadappa, [1] proposed the advantages and disadvantages of MANETS and recent developments with applications in ad hoc networks.

The authors Wataru Toorisakay, Go Hasegawaz, and Masayuki Murata, [7] proposed a mathematical analysis of power consumption in data transmission over IEEE 802.11 based wireless multi-hop networks to investigate the effects of data rate selection on energy efficiency. The analysis revealed that power consumption can be decreased by up to $13 \%$ when the symbol error rate is comparatively small.

The authors Michael J. Neely et.al. [8] proposed a dynamic algorithm for joint compression and transmission, and proves that it comes arbitrarily close to minimizing average power expenditure, with an explicit tradeoff in average delay. The algorithm is simple to implement and does not require knowledge of probability distributions for packet arrivals or channel states.

The authors Alan C. Wu et. al, [9] proposed two algorithms in choosing how to compress the data in data compression of wireless sensor networks. A translational and rotationally invariant correlation model is presented and data compression ratios are calculated from the relationship between correlation and mutual information. The analysis of energy trade-offs in data compression is presented.

\section{ANALYSIS OF EXISTING ALGORITHMS}

\subsection{Dijkstra's algorithm}

MANET assists the users to efficiently use their devices in order to minimize the battery power consumption. The communication between two devices is not necessarily symmetric, i.e., given two devices ' $i$ ' and ' $j$ '. The device ' $i$ ' can send a message to device ' $\mathrm{j}$ ', but device ' $\mathrm{j}$ ' cannot necessarily send any message to device 'i'. Since 'i' and ' $\mathrm{j}$ ' don't necessarily have the same energy. A fixed amount of data must be sent from a source device to a destination device to determine power. A new algorithm named as Dijkstra's algorithm proposed by Abdellah Idrissi et.al, for energy management in mobile ad hoc network is designed to rectify this problem [5]. It finds the minimum of energy to consume when sending a message from the device ' $s$ ' to the device ' $d$ ' in the mobile ad hoc network. The algorithm finds all shortest paths from the source to all destinations. Initially all devices are untreated, and the algorithm ends once a device is treated at destination, and moved from the untreated set to the treated set. 


\subsection{Power control algorithm}

The wireless channel is characterized by signal strength attenuating with distance from the transmitter [6]. The commonly used power-attenuation model for decay of the signal is represented by the non-linear formula

$\mathrm{P}_{\mathrm{R}}(\mathrm{d})=\frac{\mathrm{P} T}{d \alpha}$

Where, $\mathrm{P}_{\mathrm{T}}$ is the transmitted signal strength.

$d^{\alpha}$ is the distance from the transmitter.

$P_{R}(d)$ is the amplitude of the received signal at the distance' $d$ '. 2 and 6.

$\alpha$ is the path loss factor whose value typically between

Nodes can correctly receive a packet if the strength of the packet at that node is above a certain threshold called Receive threshold. If the distance between the transmitter and receiver is such that the signal strength is above this threshold, the receiver is said to be within the transmission range of the sender. There is a lower threshold called carrier sense threshold, up to which the received signal strength is enough for the receiver to detect the packet sent, but the receiver is unable to receive it correctly. All received signals that fall between these thresholds cause the channel to be sensed busy and contribute to the interference at the receiver.

Energy is a source and non-renewable in wireless ad hoc network, energy- efficient protocol design is a key concern. The design and performance analysis of such protocols require proper modeling for the measurement of energy consumption. Feeney et al, present some results of measuring energy consumption of various network interferences. The four possible energy consumption states are identified as: transmit, receive, idle and sleep. The first two states are when the node is transmitting and receiving the packets respectively, the idle state is when node is waiting for any packet transfers, and the sleep state is a very low power state where the node can neither receive nor transmit.

The cost associated with each packet at a node is represented as the total of incremental cost ' $\mathrm{m}$ ' proportional to the packet size and a fixed cost ' $b$ ' associated with channel acquisition

Cost $=m \times$ size $+b$

Thus, the cost of a broadcast packet will be of the form

Cost broadcast $=\mathrm{m}$ send $\times$ size $+\mathrm{b}$ send $+\sum_{n \in S}(\mathrm{~m}$ recv $\times$ size $+\mathrm{b}$ recv)

Where $S=$ set of nodes with in transmission range of transmitting nodes

$\mathrm{m}_{\text {send }}, \mathrm{b}_{\text {send }}=$ incremental and fixed cost for sending the broadcast packet

$\mathrm{m}_{\text {recev }}, \mathrm{b}$ recev $=$ incremental and fixed cost for received the broadcast packet.

Similarly the cost of point-to-point traffic at the sender and receiver while considering presence of RTS/CTS control messages in IEEE 802.11 based networks can be respectively represented by the following equation:-

Cost unicast - sender $=\mathrm{b}_{\text {sendctl }}+\mathrm{b}_{\text {recvctl }}+\mathrm{m}_{\text {send }} \times$ size $+\mathrm{b}$ send $+\mathrm{b}_{\text {recvetl }}$
Cost unicast - receiver $=\mathrm{b}_{\text {recvctl }}+\mathrm{b}_{\text {sendctl }}+\mathrm{m}_{\text {recev }} \times$ size +

$\mathrm{b}_{\text {recev }}+\mathrm{b}_{\text {sendctl }}$

Where $\mathrm{b}_{\text {sendctl }}=$ fixed cost for sending a control packet

$\mathrm{b}_{\text {recvetl }}=$ fixed cost for receiving a control packet

Beside this model for energy costs, it was also shown that the energy consumed by an idle network interface dominates the total energy consumption (about a magnitude more than sleep mode).The energy spend at each node due to a flow can be calculated in a simple way.

\section{OVERVIEW OF PROPOSED ALGORITHM}

\subsection{Compression}

The LZW compression algorithm in its simplest form is shown in the figure 1 . The compression algorithm builds a string translation table from the text being compressed. The string translation table maps fixed-length codes (usually 12bit) to strings. The string table is initialized with all singlecharacter strings (256 entries in the case of 8-bit characters). As the compressor character-serially examines the text, it stores every unique two-character string into the table as a code/character concatenation, with the code mapping to the corresponding first character. As each two-character string is stored, the first character is sent out. Whenever a previouslyencountered string is read from the input, the longest such previously-encountered string is determined, and then the code for this string concatenated with the extension character (the next character in the input) is stored in the table. The code for this longest previously-encountered string is sent out and the extension character is used as the beginning of the next string.

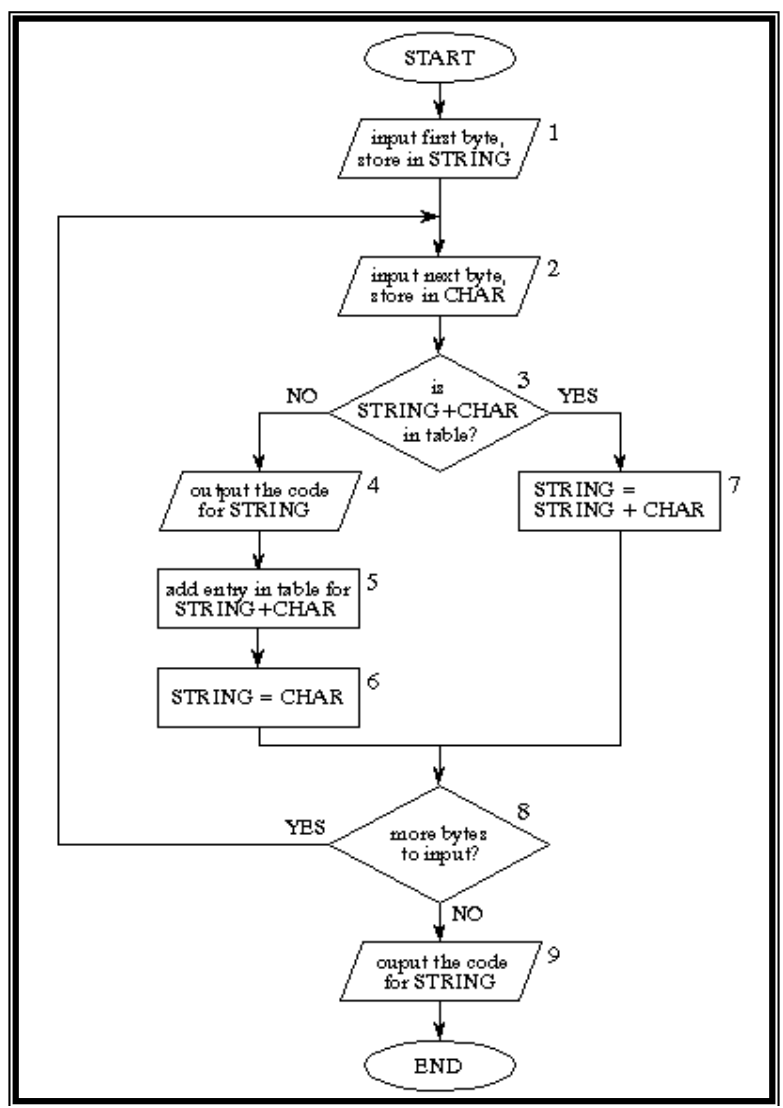

Figure 1: Compression Flowchart 


\subsection{Decompression}

The decompression algorithm needs to be able to take the stream of codes output from the compression algorithm, and use them to exactly recreate the input stream as shown in the figure 2. One reason for the efficiency of the LZW algorithm is that it does not need to pass the string table to the decompression code. The table can be built exactly as it was during compression, using the input stream as data. This is possible because the compression algorithm always outputs the STRING and CHARACTER components of a code before it uses it in the output stream. This means that the compressed data is not burdened with carrying a large string translation table.

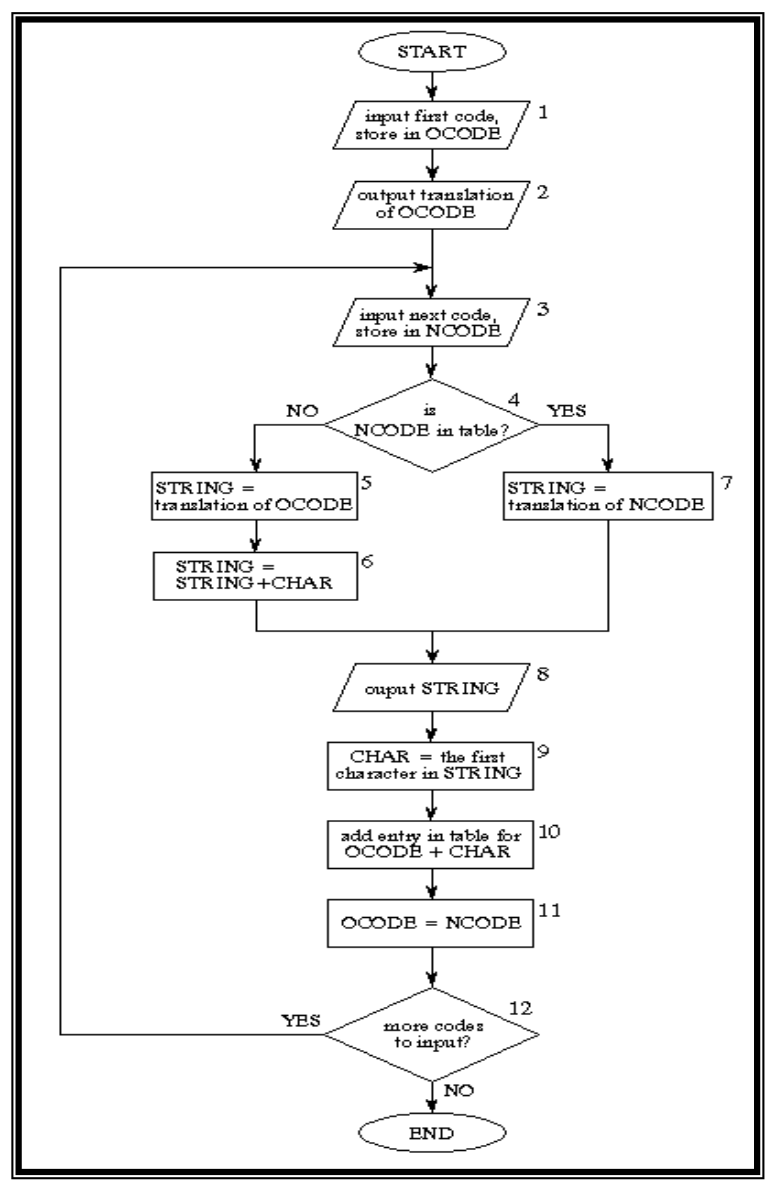

Figure 2: Decompression Flowchart

\section{IMPLEMENTATION AND SIMULATION RESULTS}

In order to evaluate the network performance of the proposed power consumption mechanism, Ad hoc Network is simulated using Global Mobile Information System Simulation (GloMoSim). It is a scalable simulation environment for large wireless network systems are uses a parallel discrete event simulation capability provided by C-based Parallel Simulation Environment for Complex System (PARSEC). The proposed method was simulated considering the following parameters as shown in table 2 .
Table 2. Simulation parameters

\begin{tabular}{|c|c|}
\hline Parameter & Value \\
\hline Simulation area & 2000 X 2000 \\
\hline Number of Nodes & 30 \\
\hline MAC Protocol & 802.11 \\
\hline Transport layer & UDP and TCP \\
\hline Traffic Generator & CBR \\
\hline Mobility & Random way point \\
\hline Node placement & Random \\
\hline Routing Protocol & AODV \\
\hline Simulation time & $500 s$ \\
\hline Bandwidth & 2 Mbps \\
\hline
\end{tabular}

The LZW algorithm is implemented in the GloMoSim with the following metrics to evaluate the efficiency in addition to the effectiveness of the algorithm.

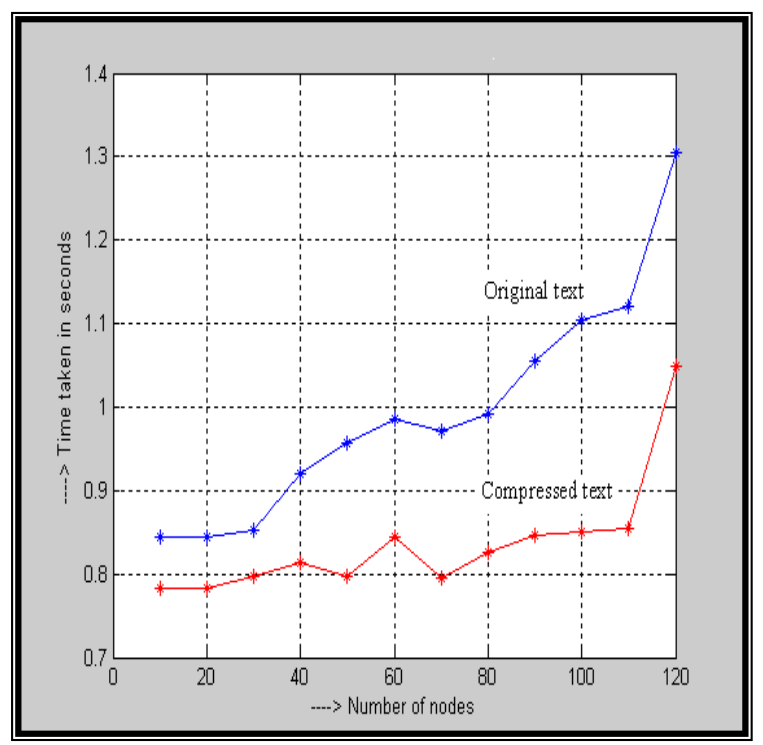

Fig3: Numbers of Nodes vs. Time

Figure 3 shows, the numbers of nodes through which the text is sent is plotted in the $\mathrm{x}$ - axis, where as time taken ('seconds') to transmit the specified data from the source node to destination node is plotted in the y-axis. It is observed that as the number of nodes increases, the time taken to transmit data packets is high in the case when the compression technique is not used. With the implementation of LZW compression algorithm, it is observed that the time taken to transmit the data packets is much less. Therefore the figure 3 concludes that by using the LZW compression algorithm, the transmission delay can be minimized. 


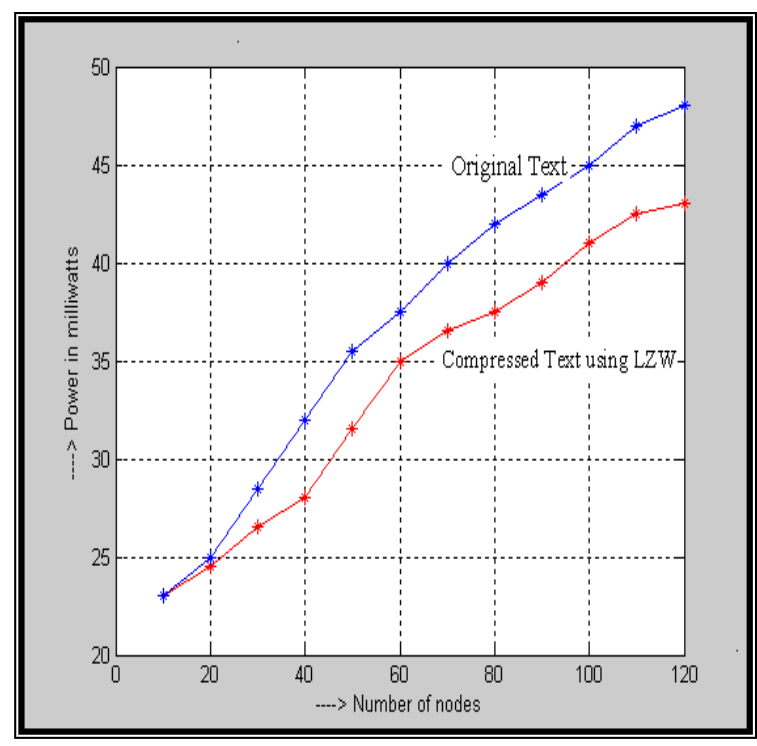

Fig 4: Number of Nodes vs. power

Figure 4 shows, the number of nodes through which the text data is transmitted is plotted in the $\mathrm{x}$-axis, where as power consumed (in 'MilliWatts') in transmitting the specified data is plotted in the y-axis. Different data packets, of different sizes are sent through the different nodes in the network. The power consumed for transmitting the data from the source to destination is noted down. It is observed that when the original data bits are transmitted without any compression, it requires more power. By using LZW algorithm, the power required to transmit the data is less.

It is observed from the result analysis, after implementing the LZW compression, data could be sent with reduced power consumption without any loss of data. This in turn helps in reducing the battery consumption, thereby increasing battery life.

\section{CONCLUSION AND FUTURE WORK}

In this work, LZW algorithm which is efficient for compression is implemented and simulated successfully in the Mobile Ad hoc Networks using GLOMOSIM. With this compression technique, any text or document file can be compressed to a maximum of one-third of its original size without any loss of data and the performance of the algorithm is analyzed before and after compression. It is observed that LZW algorithm yields high compression ratio only for the huge text and the power consumption has been reduced which reflects increase in battery life.

In future, other attributes in this limited battery life can be addressed, so that the Quality of Service in Mobile Ad hoc
Networks can be improved. In the future, image and video compression can also be implemented in Mobile Ad hoc Networks with efficient algorithms aiming minimization of power consumption.

\section{REFERENCES}

[1] Subir kumar sharkar, T.G.Basavaraju, C.Puttamadappa, "Ad hoc mobile wireless networks", Principles, protocols and applications, Auerbach Publications Boston, MA, USA @2007.

[2] Ravindra .E, VinayaDatt V Kohir and V. D Mytri, "A local route repair algorithm based on link failure prediction in mobile ad hoc network", World Journal of Science and Technology 2011.

[3] Laura marie feeney, "Energy-efficient communication in ad hoc wireless networks", Ad hoc Mobile Wireless Networks, chapter 11, Page no.314 - 320.

[4] Diaa Salama, Hatem Abdual Kader, and Mohiy Hadhoud, "Studying the effects of Most Common Encryption Algorithms", International Arab Journal of eTechnology, Vol. 2, No. 1, January 2011.

[5] Abdellah Idrissi, "How to minimize the energy consumption in mobile ad-hoc networks", International Journal of Artificial Intelligence \& Applications (IJAIA), Vol.3, No.2, March 2012.

[6] Géraud Allard, Pascale Minet, Dang-Quan Nguyen, Nirisha Shrestha, "Evaluation of the Energy Consumption in MANET", Institute national de recherché en informatique et en automatique, N 5851, FEBRUARY 2006.

[7] Wataru Toorisakay, Go Hasegawaz, and Masayuki Murata, "Power Consumption Analysis of Data Transmission in IEEE 802.11 Multi-hop Networks", The Eighth International Conference on Networking and Services, ICNS 2012.

[8] Michael J. Neely, "Dynamic Data Compression for Wireless Transmission over a Fading Channel", University of Southern California, http://wwwrcf.usc.edu/_mjneely.

[9] Alan C. Wu, "Power Efficiency with Data Compression in Wireless Sensor Networks", https://hkn.eecs.berkeley.edu.

[10] B.Ruxanayasmin, Dr. B.Ananda Krishna, "Minimization of power consumption in MANETs", International journal of Engineering Research and Technology (IJERT), PP.31-35, 2013. 